\title{
Results on top physics by CMS
}

\author{
Silvano Tosi ${ }^{1,2, a}$, on behalf of the CMS Collaboration \\ ${ }^{1}$ Università degli Studi di Genova, via Dodecaneso 33, 16146 Genova, Italy \\ ${ }^{2}$ INFN Sezione di Genova, via Dodecaneso 33, 16146 Genova, Italy
}

\begin{abstract}
In this talk, several measurements in the top-quark sector are presented, obtained from the CMS data collected in 2011 and 2012 at centre-of-mass energies of 7 and $8 \mathrm{TeV}$. The production cross section of $t \bar{t}$ pairs is measured in several decay channels: lepton+jets, dilepton and fully hadronic channels, including channels with the $\tau$ lepton. Differential cross sections are measured and are provided as a function of various kinematic observables; the results are compared with precise theory calculations. Measurements of several top-quark properties are reported, including the top pair charge asymmetry, the $\mathrm{W}$ helicity in top decays, the measurement of the production of $t \bar{t}$ pairs with $\mathrm{W}, \mathrm{Z}$, gamma, the spin correlation and the search for anomalous couplings and flavor-changing neutral currents. The results are compared with predictions from the standard model as well as new physics models. The cross sections for the electroweak production of single top quarks are measured in the t-channel and in association with W-bosons. Measurements of top-quark properties in single top quark production are also presented. The results include the W-helicity in top quark decays and the searches for the s-channel production and anomalous couplings.
\end{abstract}

\section{Introduction}

The top quark has a peculiar place in the Standard Model (SM) of particle physics. It is the heaviest elementary particle known to date, its mass being very close to the scale of electroweak symmetry breaking. As such it plays a special role in many models of new physics (NP) beyond the SM.

In the SM, the top quark decays almost exclusively to a $W b$ pair. At the CERN Large Hadron Collider (LHC), the main production mechanism of top quarks is $t \bar{t}$ pair production, via the strong interaction: the total cross-section is around $250 \mathrm{pb}$ at a center-of-mass energy of $8 \mathrm{TeV}$ and $172 \mathrm{pb}$ at $7 \mathrm{TeV}$, at the NLO. The top quark can also be produced via the electro-weak interaction, the so-called "single-top": three possible channels exist with a total cross section of around $115 \mathrm{pb}$ at $8 \mathrm{TeV}$ and 79 $\mathrm{pb}$ at $7 \mathrm{TeV}$, at the NLO.

From an experimental point of view, $t \bar{t}$ pairs can be classified in three channels according to the decay of the two $W$ bosons originated by the top and anti-top decays: dileptonic channel, when both $W$ decay leptonically; all-hadronic channel, when both $W$ decay to quarks; semi-leptonic channel, when one $W$ decays to leptons and the other to quarks. The three channels have a branching fraction of about 10, 48 and $42 \%$, respectively. Owing to the very different experimental reconstruction of

\footnotetext{
ae-mail: tosi@ge.infn.it
} 
$\tau$ leptons with respect to muons and electrons, leptonic decays comprising the $\tau$ are typically subclassified separately.

The top quark has a paramount importance at the LHC. Final states originated from top decays typically include jets, leptons and missing energy, hence involve almost all CMS sub-detectors and allow thorough tests of the performances of the detector. Moreover, studies of top-quark production and properties represent an important tool to verify SM predictions and QCD calculations in the LHC environment.

In addition, several extensions of the SM foresee a preferential coupling to the third generation and in particular to the top-quark sector, therefore top physics is a privileged window to NP.

CMS has a very rich program of measurements of top-quark properties: a selection of recent results was reported in this talk.

\section{Ingredients for top-quark physics at CMS}

The LHC started to deliver $p p$ collisions at the end of 2009. Following two periods of data taking at a $p p$ center-of-mass energy of $900 \mathrm{GeV}$ and $2360 \mathrm{GeV}$, during years 2010 and 2011 collisions at $7 \mathrm{TeV}$ have been recorded, and in 2012 the center-of-mass energy was raised to $8 \mathrm{TeV}$. The total integrated luminosity delivered by the LHC to CMS is about $6 \mathrm{fb}^{-1}$ at $7 \mathrm{TeV}$ and $23 \mathrm{fb}^{-1}$ at $8 \mathrm{TeV}$.

The collisions produced by the LHC are recorded with high efficiency by the CMS detector, which is described in detail elsewhere [1].

The reconstruction of final states of top decays typically involves several basic objects at the same time: leptons, jets, including b-tagged jets, missing energy.

Jets and missing energy are reconstructed using the "particle flow" algorithm, where the information from all sub-detectors is combined to determine the particle content of the events. This greatly improves on the performances of jet identification and resolution with respect to a reconstruction only based on energy deposits in the calorimeters. The energy scale uncertainty for jets typically varies between 1 and $3 \%$ depending on $E_{T}$, the transverse energy of the jets.

In events containing top pairs, two jets are originated from the hadronization of a $b$ quark. Several algorithms have been developed to identify the $b$-flavor of jets, which exploit various properties of $b$-hadrons, for instance the presence of a displaced secondary vertex or of secondary non-isolated leptons. These algorithms cover a broad range of signal efficiency and mis-identification rates and are chosen for each data analysis according to the specific needs.

\section{$3 t \bar{t}$ cross-section measurements}

The inclusive cross section for $t \bar{t}$ pair production was measured using basically all the possible decay topologies [2]. The results both at 7 and $8 \mathrm{TeV}$ are shown in Fig. 1: the cross section was found to be consistent with the theoretical calculations. The combination between the various measurements and with the results by the ATLAS experiment are typically performed using the BLUE algorithm [3]; a working group has been set up [4] to calculate the combination in the most appropriate way, taking into account the correlations and providing guidances on how to perform the measurements so as to make the combinations easier in the future, harmonizing the treatment of the systematic uncertainties, the choice of the theoretical parameters in the simulations etc.

The $t \bar{t}$ cross section can also be used to extract the top pole mass and the value of $\alpha_{s}$ at the $Z$-mass scale [5]. In fact, the cross section depends on both parameters: by constraining one of the two to its known value, one can measure the other one. With this method, the top pole mass was measured to be $176.7_{-2.8}^{+3.0} \mathrm{GeV}$ and $\alpha_{s}$ was measured to be $0.1151_{-0.0027}^{+0.0028}$. 

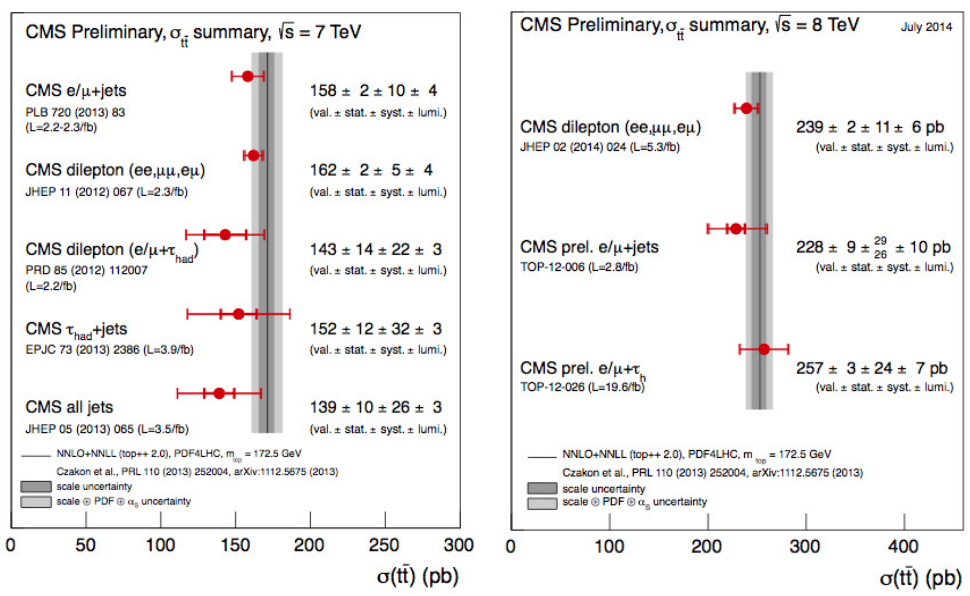

Figure 1. CMS $t \bar{t}$ inclusive cross section measurements at $7 \mathrm{TeV}$ (left) and $8 \mathrm{TeV}$ (right).

It is also important to measure the $t \bar{t}$ differential cross section as a function of various quantities because, while the inclusive cross section may be in agreement with the SM expectations, still NP can hide itself in the tails of the distributions [6]. Differential cross section measurements were reported with both the lepton+jets and the dilepton channels as a function of many observables of the reconstructed top candidates, of the top pairs and also of the global event, and were compared to predictions of several Monte Carlo generators and approximate NNLO theoretical calculations. In all cases, good agreement with SM predictions was observed.

The cross sections of $t \bar{t}$ pairs produced in association with bosons provide an additional important test of SM predictions [7]. The SM foresees a very small cross section for the $t \bar{t}+W$ and $t \bar{t}+Z$ processes. Summing up 9 different final state topologies, CMS reported an evidence for $t \bar{t}+V$ (where $V$ is either a $W$ or a $Z$ boson) with a significance of around 3.7 standard deviations $(\sigma)$; the cross section was measured to be $380_{-90}^{+100}(\text { stat })_{-70}^{+80}($ syst $) \mathrm{fb}$, consistent with the SM expectations. The $t \bar{t}+\gamma$ process was also observed with a cross section of $2.4 \pm 0.2 \pm 0.6 \mathrm{pb}$, consistent with the SM expectation of $\sim 1.8 \mathrm{pb}$.

Another very rare process in the SM is $t \bar{t}+t \bar{t}$, with an expected cross section of around $1 \mathrm{fb}$, at the $\mathrm{LO}$ at $8 \mathrm{TeV}$. Various NP scenarios could enhance this value. CMS sought for the 4-top final state [8] and observed no signal reporting an upper limit on the cross section of $63 \mathrm{fb}$ at the $95 \%$ C.L.. A similar final state topology characterizes the $t \bar{t}+b \bar{b}$ process [9]: this is not a rare process in the SM; a precise measurement of its cross section is of help in searches of associated Higgs boson production and $t \bar{t}$ pairs where of course $t \bar{t}+b \bar{b}$ represents an irreducible background if the Higgs decays to $b \bar{b}$ pairs. The $t \bar{t}+b \bar{b}$ cross section was measured relative to $t \bar{t}+2$ jets in various kinematic regions, greatly improving on the previous precision.

\section{Single top}

The electroweak production of top quarks, also called single top, can proceed via 3 kinds of diagrams, yielding the so-called $t, t W$ and $s$ channels. At $8 \mathrm{TeV}$, at an approximate NNLO, the $t$ channel has an expected cross section of around $87.2 \mathrm{pb}$, the $t W$ channel of around $22.2 \mathrm{pb}$ and the $s$ channel 5.55 $\mathrm{pb}$. Notice that at the Tevatron, in $p \bar{p}$ collisions, the $s$ channel has instead a much larger cross section with respect to the $t W$ channel. 
CMS measured the cross section of the $t$ channel to be $83.6 \pm 2.3 \pm 7.4 \mathrm{pb}$ at $8 \mathrm{TeV}$, consistent with the SM [10]. Also, the charge ratio $\sigma(t) / \sigma(\bar{t})$ was measured which is sensitive to the various parton distribution functions [10].

The $t W$ channel was observed for the first time by CMS with a cross section of $23.4 \pm 5.4 \mathrm{pb}$ [11]. The $s$ channel was not observed yet and an upper limit on the cross section was reported of $11.5 \mathrm{pb}$ at the $95 \%$ C.L. [12].

\section{Top-quark properties}

The mass of the top quark is a fundamental parameter within the SM and affects predictions of SM observables via radiative corrections. Several methods have been developed to measure the top-quark mass. CMS used improved versions of the matrix weighting technique [13] and the fully kinematic method [14]. The top mass was measured by CMS with the dilepton, lepton+jets and all-hadronic channels [15]. The latest CMS combination of the top mass measurements yields the value (172.2 \pm $0.1 \pm 0.7) \mathrm{GeV}$, which will be updated soon with more results: this already represents a precision measurement. The comparison of the CMS mass measurements to those reported by ATLAS and by the Tevatron experiments [16] can be seen in Fig. 2.

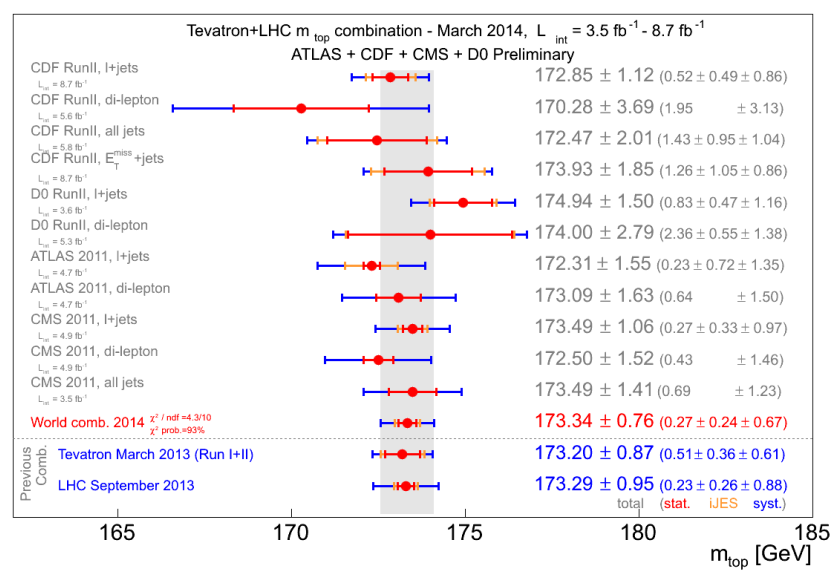

Figure 2. Top mass measurements from the Tevatron and LHC experiments.

When the uncertainty on the mass reaches values below $\sim 1 \mathrm{GeV}$, the interpretation of the reconstructed mass becomes not trivial because the top is an unstable, colored object and ambiguities arise due to the color flow in the event. For this reason, the dependence of the measured mass value on several kinematic variables was studied and no deviation with respect to the predictions of various generators was yet observed [17].

CMS also measured the mass difference between the top and the anti-top, which provides a test of CPT invariance [18]. The difference was measured to be consistent with the null expectation: $-272 \pm 196 \pm 122 \mathrm{MeV}$.

The spins of the top and the anti-top in $t \bar{t}$ pair production are correlated in the SM, and the leptons from the decays of the $W$ bosons carry information on the spin history of the event. CMS measured the differential cross section of top pair production as a function of angular variables of the leptons and found patterns consistent with the SM [19]. This allows one to constrain NP contributions that could alter the prediction: for example, we can probe anomalous $g t t$ couplings with an effective field theory 
approach. Limits on the real part of the chromo-magnetic dipole moment of the top were obtained: $-0.043<\operatorname{Re}\left(\mu_{t}\right)<0.117$ at the $95 \%$ C.L. [20].

In the SM, top pairs are produced in a symmetric state, as far as the color charge is concerned, at the LO. At the NLO, a small asymmetry is present, which can be enhanced in various NP scenarios, for example if the $t \bar{t}$ pair is originated by the decay of a heavy resonance. At the Tevatron experiments, where the initial state is $p \bar{p}$, a charge asymmetry yields a forward-backward asymmetry: the Tevatron experiments indeed reported a deviation of the forward-backward asymmetry from SM expectations by around 2 standard deviations [21]. At the LHC, where the initial state is $p p$, a charge asymmetry can induce a difference of absolute pseudo rapidities of top and anti-top quarks, $\left|\eta_{t}\right|-\left|\eta_{\bar{t}}\right|$. Using both dileptonic and semileptonic $t \bar{t}$ pairs, CMS measures a null asymmetry, consistent with the SM expectations [22]. CMS also reported a differential measurement of the charge asymmetry and again, no deviation with respect to the SM was seen in any corner of the phase space.

The ratio $R=B R(t \rightarrow W b) / B R(t \rightarrow W q)$ is basically 1 in the SM and, in a 3-generation scenario, it equals $\left|V_{t b}\right|^{2}$. CMS measured a value of $R$ of $1.014 \pm 0.003 \pm 0.032$ consistent with the SM [23], whereas the D0 experiment reported some tension, especially in the dileptonic channel $(0.86 \pm 0.04 \pm 0.04$ [24]). The measurement of $R$ translates into a constraint on $\left|V_{t b}\right|$ to be greater than 0.975 at the $95 \%$ C.L. and also provides a measurement of the width of the top: $\Gamma($ top $)=1.36 \pm 0.02_{-0.11}^{+0.14} \mathrm{GeV}$.

While in the SM only a left vector coupling is present in the $W t b$ vertex, right vector couplings and left and right tensor couplings could appear in NP scenarios. 95\% C.L. upper limits on the right vector coupling and on the left tensor coupling were obtained of 0.34 and 0.09 , respectively.

If we define $\theta^{*}$ as the polar angle of the lepton from the $W$ decay in the $W$ rest frame, the differential decay width can be written as

$$
\frac{1}{\Gamma} \frac{d \Gamma}{d \cos \theta^{*}}=\frac{3}{8}\left(1-\cos \theta^{*}\right)^{2} F_{L}+\frac{3}{8}\left(1+\cos \theta^{*}\right)^{2} F_{R}+\frac{3}{4}\left(\sin \theta^{*}\right)^{2} F_{0}
$$

where $F_{0}, F_{L}$ and $F_{R}$ represent the longitudinal, left and right helicity fractions of the $W$ boson. These are calculated to be $0.69,0.31$ and $<0.01$ in the SM, respectively. CMS measured them with both $t \bar{t}$ pairs and single top, and obtained values consistent with the SM [25]. This in turn allows to constrain NP models where the $W t b$ couplings could be modified with respect to the SM.

Flavor-changing neutral currents (FCNC) are transitions from a quark to a different-flavor quark of the same charge, for example $t \rightarrow u \gamma$. These are forbidden at the tree level in the SM, and are only possible via higher-order diagrams, which are strongly suppressed, yielding branching fractions of FCNC top decays in the range $10^{-13}-10^{-11}$. NP can modify the picture, enhancing some of these processes if for instance new particles enter the loops. CMS sought for FCNC processes both using $t \bar{t}$ and single top events and reported no evidence for them [26]: upper limits on various decay modes were obtained, which in turn allow to start constraining NP models.

\section{Conclusion}

The CMS experiment has already reported several interesting results on top-quark physics. So far, no departure from the SM predictions was seen, and, thanks to the large data sample provided by the LHC, several results already are precision measurements. A rich program of study of inclusive and differential distributions and properties of the top quark is in place. More results on the 7 and $8 \mathrm{TeV}$ data are on the way and new results will soon be obtained at higher center-of-mass energies with the new LHC data to come in the next years. 


\section{References}

[1] CMS Collaboration, Journal of Instrumentation 0803 (2008)

[2] CMS Collaboration, TOP-12-026, https://cds.cern.ch/record/1745684; Journal of High Energy Physics 02, 024 (2014); Journal of High Energy Physics 1305, 065 (2013); European Physics Journal C73, 2386 (2013); Physics Letters B 720, 83 (2013)

[3] L. Lyons, D. Gibaut and P. Clifford, Nuclear Instruments and Methods A 270, 110-117 (1988); A. Valassi, Nuclear Instruments and Methods A 500, 391 (2003)

[4] The Top Physics LHC Working Group, https://twiki.cern.ch/twiki/bin/view/LHCPhysics/TopLHCWG

[5] CMS Collaboration, Physics Letters B 728, 496 (2013)

[6] CMS Collaboration, TOP-12-027, https://cds.cern.ch/record/1523611; TOP-12-028, https://cds.cern.ch/record/1523664

[7] CMS Collaboration, European Physics Journal C74 3060 (2014); TOP-13-011, http://cds.cern.ch/record/1644573; Physical Review Letters 110172002 (2013)

[8] CMS Collaboration, TOP-13-012, http://cds.cern.ch/record/1644574

[9] CMS Collaboration, TOP-13-010, http://cds.cern.ch/record/1605842; TOP-12-024, http://cds.cern.ch/record/1479150

[10] CMS Collaboration, Journal of High Energy Physics 06, 090 (2014)

[11] CMS Collaboration, Physical Review Letters 112, 231802 (2014)

[12] CMS Collaboration, TOP-13-009, https://cds.cern.ch/record/1633190

[13] D0 Collaboration, Physical Review Letters 802063 (1998)

[14] CDF Collaboration, Physical Review D73 112006 (2006)

[15] CMS Collaboration, European Physics Journal C74, 2758 (2014); TOP-14-002 http://cds.cern.ch/record/1743141;TOP-14-001, http://cds.cern.ch/record/1690093

[16] CMS Collaboration, TOP-13-014, http://cds.cern.ch/record/1669819

[17] CMS Collaboration, TOP-12-029, https://cds.cern.ch/record/1521357

[18] CMS Collaboration, TOP-12-031, https://cds.cern.ch/record/1528156

[19] CMS Collaboration, Physical Review Letters 112182001 (2014)

[20] CMS Collaboration, TOP-14-005, http://cds.cern.ch/record/1745175

[21] D0 Collaboration, Physical Review Letters 100, 142002 (2008); CDF Collaboration, Physical Review D87, 092002 (2013)

[22] CMS Collaboration, Journal of High Energy Physics 04, 191 (2014)

[23] CMS Collaboration, Physics Letters B 736, 33 (2014)

[24] D0 Collaboration, Physical Review Letters 107, 121802 (2011)

[25] CMS Collaboration, Journal of High Energy Physics 10, 167 (2013); TOP-12-020, https://cds.cern.ch/record/1523619

[26] CMS Collaboration, Physical Review Letters 112, 171802 (2014) ; TOP-14-007, http://cds.cern.ch/record/1702400; TOP-14-003, http://cds.cern.ch/record/1700519 